Major reasons for studying the inner corona (apart from its intrinsic astrophysical interest) are that it probably holds the key to a detailed understanding of the solar (and stellar) winds, and because it controls the immediate environment of the Earth in space. It is therefore pertinent to review here briefly what is needed. First, we want to know in more detail the temperature structure of the inner corona. We have seen that an average model for the low corona in active regions, quiet regions and holes is emerging from the EUV data. However, the height in the corona where the temperature reaches a maximum is not known and is difficult to determine. Further it is now clear that the temperature of the inner corona is not homogeneous. We have seen that there is indirect evidence that the corona varies from SGCs to SGBs. The ATM experiment has the resolution to throw much light on this problem. Finally, we require the deposition of energy by waves, as a function of height in the inner corona. Here we have to rely heavily on theory, but much progress has been made on the problem of the energy balance - at least in the transition region and low corona (Athay, 1971).

\title{
WORKING GROUP ON THE HIGH-RESOLUTION ATLAS \\ OF THE PHOTOSPHERIC SPECTRUM
}

Tables of wavelengths and identifications covering the domain $\lambda 7498-\lambda 12016$ have been published recently (Swensson et al., 1970). Based on the Atlas published in 1963 by L. Delbouille and G. Roland, this work lists 10840 lines, and gives identifications for a large fraction of them. A highresolution, low-noise atlas of the photospheric spectrum between 3000 and $10000 \AA$ has been announced by Delbouille, Roland and Neven. The observations have been made from the highaltitude station of the Jungfraujoch (Switzerland), to minimize the water-vapour absorption. The section $\lambda 4300-\lambda 6200$ will be distributed first, and the subscribers will receive shorter and longer wavelength tracings with the work progressing.

A preliminary atlas, designed to supplement the above-mentioned work, is also in preparation at Kitt Peak National Observatory. The complete spectrum has been run for $\cos v=1.0$, and about $40 \%$ has been completed for $\cos v=0.2$. At the request of stellar observers, selected portions of the integrated solar spectrum have been observed.

The photographic wavelength program $(\lambda 2950-\lambda 10000)$, started by A. K. Pierce in 1967 , is nearly complete; it should be finished by the end of 1973 . The necessary standard wavelengths, against which the solar values are measured, are given by a thorium source, in overlapping orders. Unblended lines are measured by hand on the plates and reduced by computer. Approximately 10 spectra are combined into each wavelength measurement, and the internal accuracy is about $0.5 \mathrm{~m} \AA$ for many lines. The photoelectric part of the same program, with computer simulation of the blended profiles to resolve them, has just started.

L. DELBOUILLE

Chairman of the Working Group

\section{WORKING GROUP ON SUNSPOT SPECTRA}

Several sets of observational data on sunspots exist:

(1) the photographic observations of R. Michard at Pic-du-Midi and Meudon on three spots covering the total range $\lambda \lambda 4900-6450$;

(2) the photoelectric tracings of $H$. Wöhl $\lambda \lambda 4000-8000$ obtained at Locarno for three large umbras;

(3) a photographic map with Babinet compensator of one large spot covering the interval $\lambda \lambda 3800-9200$ by J. Harvey at Kitt Peak;

(4) the thesis work of D. Hall giving photoelectric tracings from Kitt Peak in the atmospheric windows between 1 and $5.5 \mu \mathrm{m}$; 